\title{
A case of pulmonary edema: The critical role of lung-heart integrated ultrasound examination
}

\author{
Alfonso Sforza1, Maria Viviana Carlino1, Mario Guarino1, Salvatore Russo1, Giovanni Albano², \\ Fiorella Paladino 3 , Costantino Mancusi² \\ 1 Emergency Department, CTO Hospital, Naples \\ 2 Hypertension Research Center, UOC Emergency Medicine, Federico II University Hospital, Naples \\ 3 Emergency Department, Cardarelli Hospital, Naples, Italy
}

\begin{abstract}
Cardiogenic pulmonary edema is a common presentation of acute heart failure normally treated with continuous positive airway pressure (CPAP), diuretics and nitrates. This therapy is contraindicated in case of cardiac tamponade. We describe a case of pulmonary edema due to cardiac tamponade in which integrated lung-heart ultrasound examination allowed prompt diagnosis and pericardiocentesis before administration of CPAP thus avoiding circulatory collapse.
\end{abstract}

\section{Introduction}

Pulmonary edema is one of the clinical presentations of acute heart failure and reflects the accumulation of fluid in the lung interstitium and alveoli as a result of increased capillary hydrostatic pressure. This is the result of an increase in left ventricular filling pressures secondary to diastolic dysfunction. This condition is common in both heart failure with reduced and preserved ejection fraction. Acute coronary syndromes, hypertensive emergencies, cardiomyopathies, valvular dysfunctions may lead to acute decompensated heart failure and pulmonary edema. Integrated lung-heart-inferior vena cava ultrasound examination allows prompt diagnosis and can guide therapy by identification of different hemodynamic patterns [1,2]. Lung ultrasound provides estimation of in-

Corresponding author: Dr. Alfonso Sforza, Emergency Department CTO Hospital, AORN dei Colli, Viale Colli Aminei 21, 80141 Naples, Italy. Tel. +39.3897837318. E-mail: alfosfo@hotmail.it

Key words: Multi-organ ultrasound; point-of-care ultrasound; acute dyspnea; heart failure; cardiac tamponade.

Contributions: AS, MVC, study concept, manuscript drafting; MG, SR, GA, FP, CM, contribution to the discussion, manuscript editing.

Conflicts of interest: The Authors declare no conflict of interest.

Received for publication: 18 July 2018

Accepted for publication: 19 September 2018

CC Copyright A. Sforza et al., 2018

Tipografia PI-ME Editrice, Italy

Monaldi Archives for Chest Disease 2018; 88:982

doi: 10.4081/monaldi.2018.982

This article is distributed under the terms of the Creative Commons Attribution Noncommercial License (by-nc 4.0) which permits any noncommercial use, distribution, and reproduction in any medium, provided the original author(s) and source are credited. terstitial lung edema [3]. Cardiac ultrasound provides information on left ventricular systolic function, chambers dimensions, valvular dysfunctions, pericardial diseases and estimation of left ventricular filling pressures [4]. Evaluation of inferior vena cava dimension and collapsibility provides estimation of central venous pressure [5].

\section{Case Report}

A 61-year-old man with history of gastroesophageal reflux disease in proton pump inhibitor therapy was transported to the emergency department for severe dyspnea. He had history of cigarette smoking and he was not affected by any cardiorespiratory disease. He referred recent fever and chest pain with diagnosis of bronchitis currently on antibiotic and anti-inflammatory therapy. At admission blood pressure was 110/70 $\mathrm{mmHg}$, heart rate was 110 beats/minute, oxygen saturation was $76 \%$ ( $\left.\mathrm{FiO}_{2} 21 \%\right)$ with tachypnea (32 breaths/minute) and body temperature was $35.7^{\circ} \mathrm{C}$. Chest examination revealed wheezing. Cardiovascular examination revealed muffled heart sounds, normal peripheral pulses, absence of leg edema and presence of jugular vein distension. Abdominal examination was normal. Arterial blood gas analysis in room air (Table 1) showed metabolic lactic acidosis with mild portion of respiratory acidosis and severe hypoxemia. Oxygen therapy with $60 \%$ Venturi mask was administrated. ECG showed sinus tachycardia with mild electrical alternans (Figure 1). B-type natriuretic peptide was $500 \mathrm{pg} / \mathrm{mL}$. An integrated lung-heart-inferior vena cava ultrasound examination with pocket ultrasound device was promptly done $[1,6]$. The integrated ultrasound examination of lung-heart-inferior vena cava was performed with the patient in semi-sitting position using a pocket size device (Vscan, General Electric Healthcare) with single probe (1.7-3.8 MHz) using abdominal pre-set for lung assessment and cardiac pre-set for heart and inferior vena cava assessment. We scanned first lungs and then heart and inferior vena cava, following the $\mathrm{ABC}$ protocol of emergency medicine [1]. Thorax was examined following a simplified protocol that provides two scans at each side: anteriorly on the II intercostal space, midclavicular line and lateral on the $\mathrm{V}$ intercostal space, midaxillary line, to sample upper and lower lungs. The heart was examined in three projections (parasternal long axis view, apical view and subcostal view) allowing qualitative evaluation of left ventricular systolic function, size of chambers and the presence or absence of pericardial effusion. The inferior vena cava was explored in subcostal view [1]. Lung ultrasound examination showed normal pleural sliding and multiple vertical comet-tail artefacts (B-lines), often confluent (white lung) and deleting horizontal artefacts (A-lines) compatible with diffuse bilateral interstitial syndrome (Figure 2) suggesting pulmonary edema. Focused cardiac examination revealed severe pericardial effusion with tamponade demonstrated by right chambers and left atrium collapse and left ventricle impaired filling (Figure 3). The pericardial effusion was circum- 
ferential with evidence of swinging heart. It was more thickened in the anterior region (more than $2 \mathrm{~cm}$ ). Right chambers were collapsed. Left atrial was partially collapsed and impaired left ventricular filling was evident. The semi quantitative assessment of pericardial effusion volume was difficult because of the evidence of swinging heart, but we can expect more than $500 \mathrm{ml}$ since it was more than $2 \mathrm{~cm}$ in 2 projections. The aortic root was normal. Inferior vena cava was dilated and fixed $(2.5 \mathrm{~cm}$ with no respiratory variation). Chest $\mathrm{X}$-ray did not give new information. The patient underwent emergency pericardiocentesis and then continuous positive airway pressure (CPAP) with prompt improvement of symptoms. CPAP was set at $10 \mathrm{cmH}_{2} \mathrm{O}$ for 6 hours. He was admitted to sub-intensive care unit and after 2 days he was transferred in the cardiology ward. He was discharged 7 days after.

The final diagnosis was cardiac tamponade due to pericarditis.

\section{Discussion}

Acute cardiogenic pulmonary edema is one of the most frequent presentation of acute heart failure. The standard therapy consists of administration of continuous positive airway pressure and medical therapy with diuretics and nitrates [7]. Cardiac tamponade is a lifethreatening condition requiring prompt recognition and immediate pericardiocentesis [8].

Pulmonary edema is quite rare during tamponade, perhaps because right heart filling is restricted, limiting pulmonary blood flow. In contrast, acute pulmonary edema has been reported after decompression of pericardial tamponade, related to the sudden increase in pulmonary blood flow [9]. However, it is described that pericardial effusion may cause interstitial pulmonary edema in experimental models. During raising of intra-pericardial pressure, all intra-cardiac and small circulation pressures (mean pulmonary arterial pressure, mean pulmonary wedge pressure, mean right ventricular pressure, microvascular hydro- static pressure, right atrial pressure) increase. The accumulation of pericardial fluid sufficient to raise pericardial pressure can create an imbalance in the Starling forces in the pulmonary vasculature that will favor increased filtration into the interstitium. The high pericardial pressure also retards pulmonary lymphatic drainage because of raising right atrial pressure thus contributing to the formation of edema [10].

CPAP exerts its effects on cardiac performance by increasing intrathoracic pressure and thereby reducing cardiac preload, by impeding cardiac filling, and afterload, by reducing left ventricular transmural pressure [11]. Nitrates induce vasodilation thus reducing preload and afterload [7]. These effects are all harmful during cardiac tamponade and could get worse the hemodynamic state with shock.

The use of multi-organ ultrasound examination in the management of acute dyspnea is becoming a cornerstone for identification of acute heart failure in the emergency department $[1,12,13]$. In this case the integrated lung-heart ultrasound examination avoided the wrong diagnostic-therapeutic strategy, allowing prompt recognition of tamponade

Table 1. Blood gas analysis in room air.

\begin{tabular}{lcc} 
Parameter & Value & Unit of measure \\
$\mathrm{pH}$ & 7.28 & \\
\hline $\mathrm{pCO}_{2}$ & 31 & $\mathrm{mmHg}$ \\
\hline $\mathrm{pO}_{2}$ & 40 & $\mathrm{mmHg}$ \\
\hline $\mathrm{Na}^{+}$ & 132 & $\mathrm{mmol} / \mathrm{L}$ \\
\hline $\mathrm{K}^{+}$ & 4.8 & $\mathrm{mmol} / \mathrm{L}$ \\
\hline $\mathrm{Ca}^{++}$ & 1.03 & $\mathrm{mmol} / \mathrm{L}$ \\
\hline $\mathrm{Glu}$ & 175 & $\mathrm{mg} / \mathrm{dL}$ \\
\hline $\mathrm{Lat}^{-}$ & 9.6 & $\mathrm{mmol} / \mathrm{L}$ \\
\hline $\mathrm{HCO}_{3}^{-}$ & 14.6 & $\mathrm{mmol} / \mathrm{L}$ \\
\hline $\mathrm{SO}_{2}$ & 76 & $\%$ \\
\hline $\mathrm{Hb}^{-}$ & 10.9 & $\mathrm{~g} / \mathrm{dL}$ \\
\hline
\end{tabular}

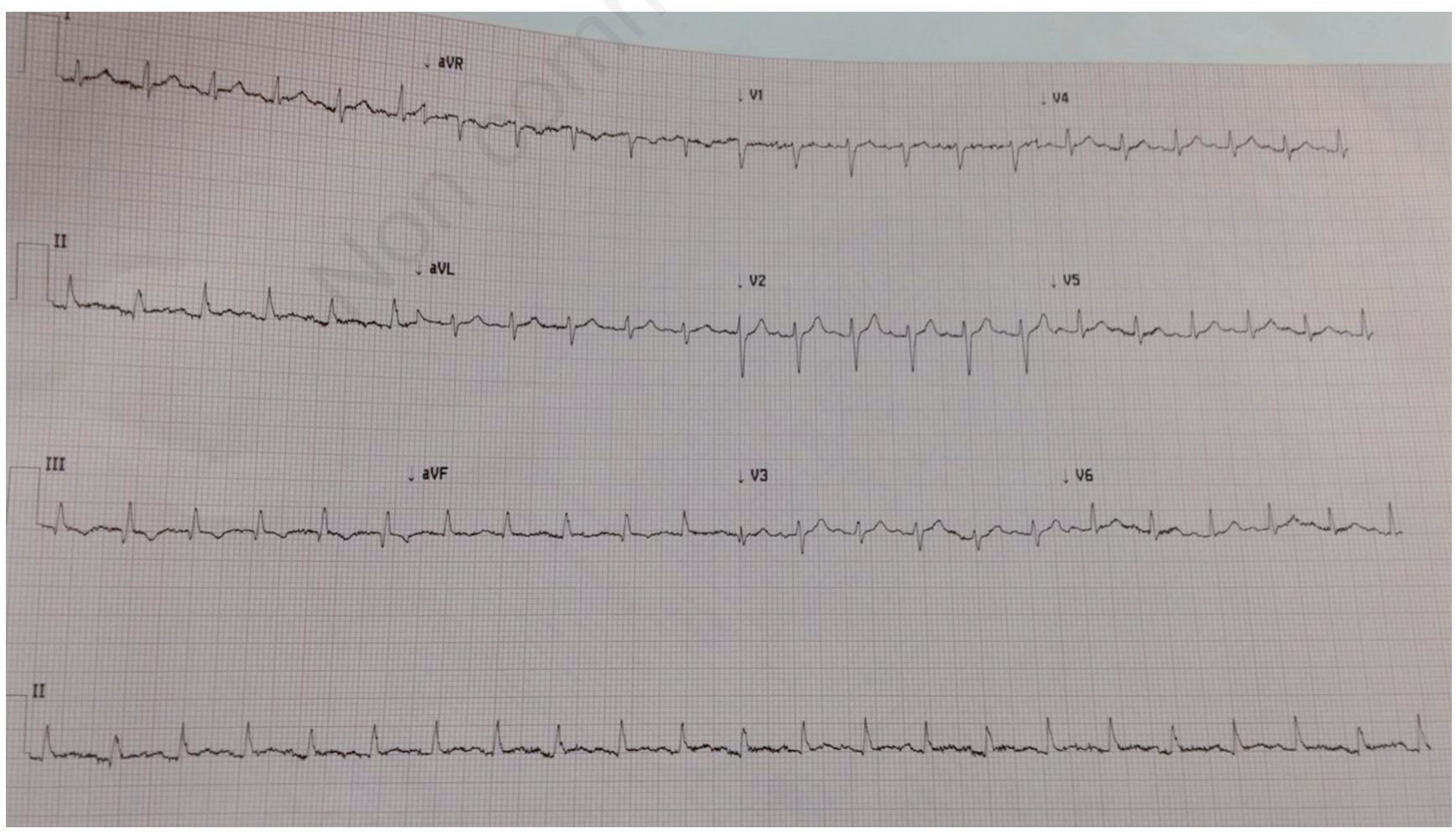

Figure 1. ECG showing sinus tachycardia and mild electrical alternans. 


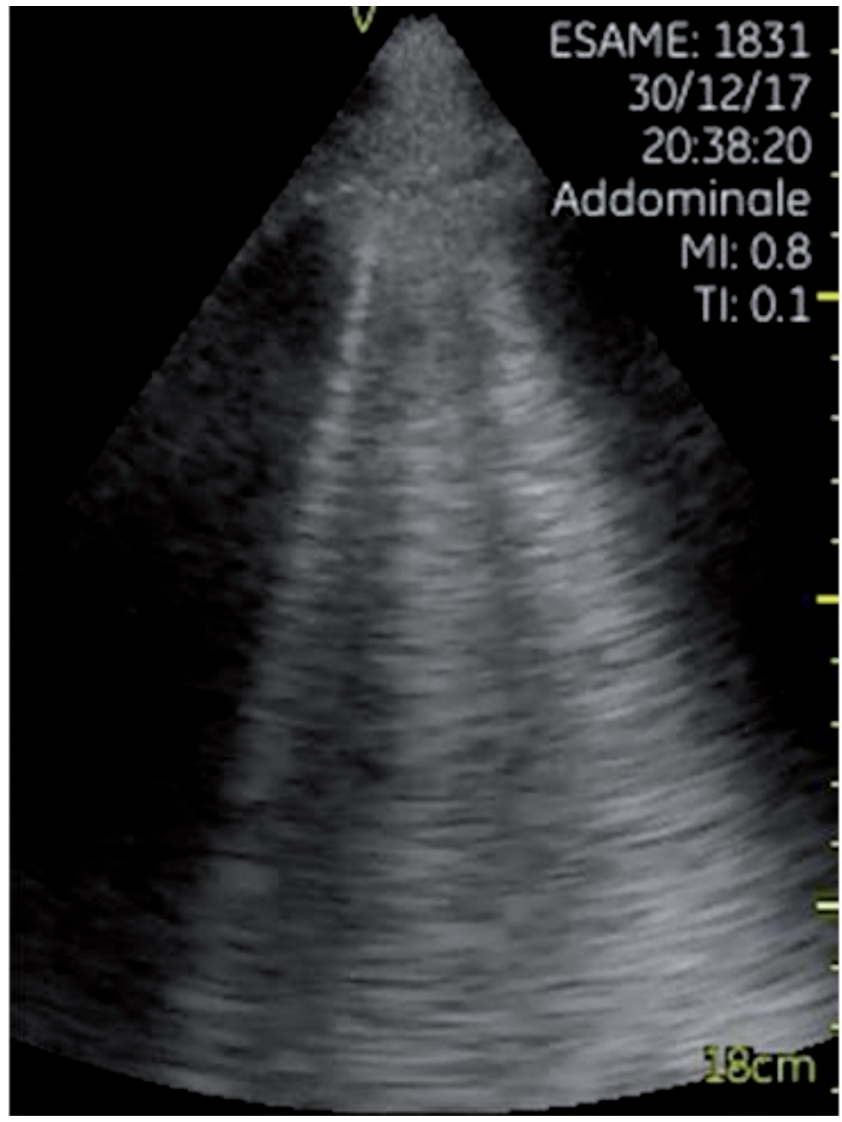

Figure 2. Lung ultrasound showing interstitial syndrome.

and the emergency pericardiocentesis before the administration of CPAP, thus preventing the downfall of hemodynamic balance. In this case, chest X-ray did not give any adjunctive information to that of lung-heart ultrasound since it showed enlarged and loss of definition of large pulmonary vessels, associated with cardiomegaly. These findings are in line with findings of fast ultrasound and did not give any new insights to the clinical pictures.

\section{Conclusions}

The integrated lung-heart ultrasound examination during acute pulmonary edema allows a more detailed diagnosis compared to the lung ultrasound alone. This is important to establish the correct sequence of therapeutic actions in patients with complex clinical pictures.

\section{References}

1. Sforza A, Mancusi C, Carlino MV, et al. Diagnostic performance of multi-organ ultrasound with pocket-sized device in the management of acute dyspnea. Cardiovasc Ultrasound 2017;15:16.

2. Almeida Junior GL, Xavier SS, Garcia MI, Clausell N. Hemodynamic assessment in heart failure: role of physical examination and noninvasive methods. Arq Bras Cardiol 2012;98:e15-21.

3. Picano E, Pellikka PA. Ultrasound of extravascular lung water: a new standard for pulmonary congestion. Eur Heart J 2016;37:2097-104.

4. Labovitz AJ, Noble VE, Bierig M, et al. Focused cardiac ultrasound in the emergent setting: a consensus statement of the American

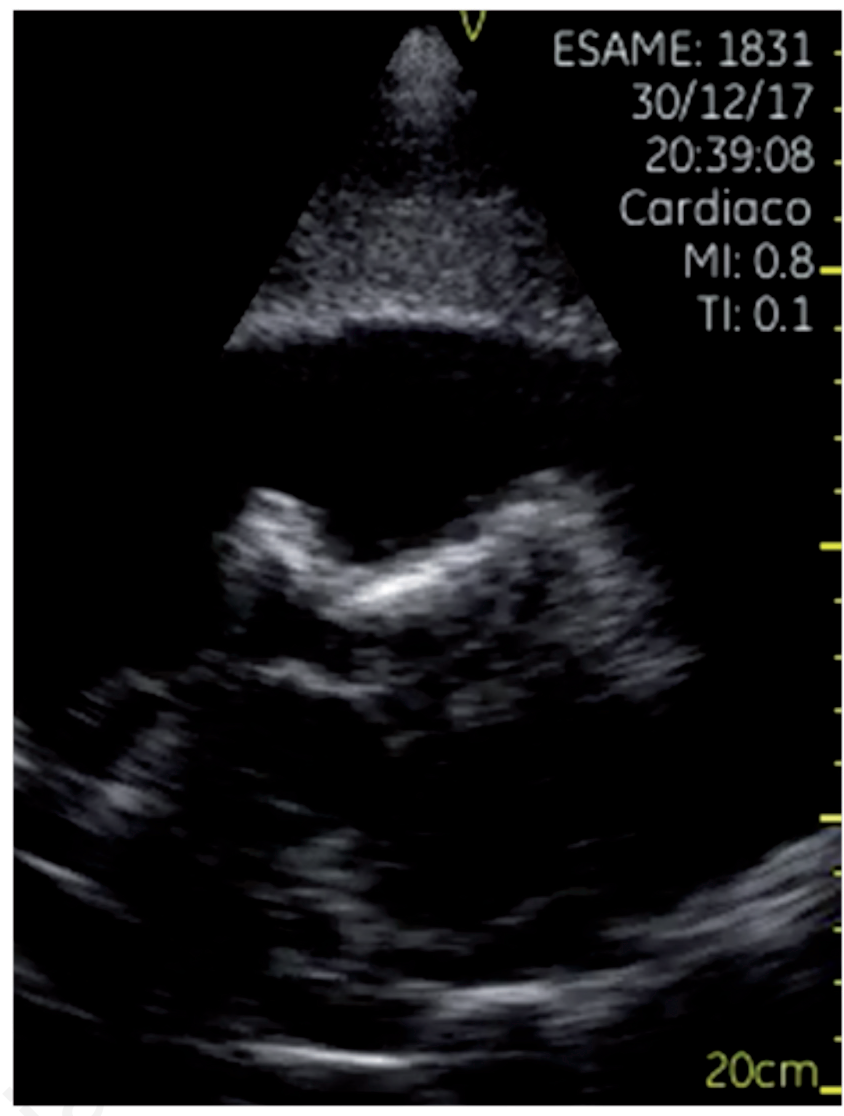

Figure 3. Echocardiography in sub-costal view showing severe pericardial effusion with right chambers collapse.

Society of Echocardiography and American College of Emergency Physicians. J Am Soc Echocardiogr 2010;23:1225-30.

5. Ciozda W, Kedan I, Kehl DW, et al. The efficacy of sonographic measurement of inferior vena cava diameter as an estimate of central venous pressure. Cardiovasc Ultrasound 2016;14:33.

6. Sforza A, Carlino MV, Romano G, Mancusi C. Detection of Stanford type A aortic dissection by focused cardiac ultrasonography performed with pocket ultrasound device in a patient with chest pain. Visual J Emergen Med 2018;11:14-15.

7. Purvey M, Allen G. Managing acute pulmonary oedema. Aust Prescr 2017;40:59-63.

8. Willner DA, Grossman SA. Pericardiocentesis. StatPearls [Internet]. November 7, 2017.

9. Vandyke WH Jr, Cure J, Chakko CS, Gheorghiade M. Pulmonary edema after pericardiocentesis for cardiac tamponade. $\mathrm{N}$ Engl J Med 1983;309:595-6.

10. Sznajder JI, Evander E, Pollak ER, et al. Pericardial effusion causes interstitial pulmonary edema in dogs. Circulation 1987;76:843-9.

11. Naughton MT, Rahman MA, Hara K, et al. Effect of continuous positive airway pressure on intrathoracic and left ventricular transmural pressures in patients with congestive heart failure. Circulation 1995;91:1725-31.

12. Carlino MV, Paladino F, Sforza A, et al. Assessment of left atrial size in addition to focused cardiopulmonary ultrasound improves diagnostic accuracy of acute heart failure in the Emergency Department. Echocardiography 2018;35:785-91.

13. Carlino MV, Mancusi C, de Simone G, et al. Interstitial syndromelung ultrasound B lines: a potential marker for pulmonary metastases? A case series. Ital J Med 2018;12:223-6. 